\title{
Heuristic Approaches for Periodic Reconfiguration of Lightpath-based Networks under Multi-hour Traffic
}

\author{
Ramon Aparicio-Pardo \\ Universidad Politécnica de Cartagena/Dept. Information and Communication Technologies, Cartagena, Spain \\ Email: ramon.aparicio@upct.es \\ Belen Garcia-Manrubia \\ Universidad Politécnica de Cartagena/Dept. Information and Communication Technologies, Cartagena, Spain \\ Email: belen.garcia@upct.es \\ Nina Skorin-Kapov \\ University of Zagreb/Faculty of Electrical Engineering and Computing (FER), Zagreb, Croatia \\ Email: nina.skorin-kapov@fer.hr \\ Pablo Pavon-Marino \\ Universidad Politécnica de Cartagena/Dept. Information and Communication Technologies, Cartagena, Spain \\ Email: pablo.pavon@upct.es
}

\begin{abstract}
Periodic lightpath reconfiguration of virtual topologies in transparent optical networks has been recently investigated as a mechanism to more efficiently adapt the network to predictable periodic traffic variations along a day or week. Scheduling periodic reconfigurations involves tuning a trade-off between a lower network cost obtained through better resource allocation, and undesired traffic disruptions that these reconfigurations may cause. This paper presents and compares two algorithms for planning a reconfigurable virtual topology suitable for exploring this trade-off. The first is based on a Lagrangean Relaxation of the planning problem, and the second is based on a Tabu Search meta-heuristic. The merits of both algorithms are assessed for moderate network sizes through comparison with analytical lower bounds and exact solutions obtained by a MILP formulation.
\end{abstract}

Index Terms-virtual topology design, network planning, multi-hour traffic, Lagrangean Relaxation, Tabu Search.

\section{INTRODUCTION}

Optical networks based on Wavelength Division Multiplexing (WDM) have become an accepted solution for today's high-speed backbone networks [1]. In such networks, traffic is carried over lightpaths. A lightpath is defined as a transparent all-optical connection between an optical transmitter in the originating node and an optical receiver in the terminating node using a wavelength channel in each traversed physical link. Since lightpaths allow bypassing the electronic processing of the traffic at intermediate nodes, savings with respect to electronic switching equipment are achieved. In order to carry a given set of traffic demands (in Gbps), a set of lightpaths, comprising a so-called virtual topology, is established over the physical topology. Therefore, the Virtual Topology Design (VTD) problem implies solving a multilayer routing problem consisting of two layers. The upper layer problem involves routing traffic flows (demands) over the virtual topology; while the lower layer problem implicates finding a Routing and Wavelength Assignment (RWA) [2] of lightpaths over the physical topology. We must note that in this work we focus only on the upper layer routing problem and we assume a feasible RWA solution exists for each lightpath in the virtual topology. This assumption can realistically depict several network scenarios, such as metro-area optical networks with an over-dimensioned fiber plant.

In this paper, we address a variant of the aforementioned VTD problem, where we assume that the traffic varies along a given period of time (e.g. a day) following a repetitive pattern, i.e., that the traffic is periodic. Therefore, the traffic demand can be represented as a temporal sequence of matrices, where each matrix is associated to a time slot. This variant is denoted as MultiHour Virtual Topology Design (MH-VTD) [3]. Real traffic traces, such as the Abilene backbone network [4] and GEANT backbone network [5], support this assumption of the periodic nature of traffic.

The MH-VTD problem can be classified into: (i) the MH-VTD-NR (non-reconfigurable) problem, where the virtual topology cannot be changed along time, which means that we determine a static set of lightpaths capable of routing all the traffic as it varies over all time slots; 
and (ii) the MH-VTD-R (reconfigurable) problem where the virtual topology changes along time to follow the multi-hour traffic variations. In both problem variants, the main objective is to minimize the number of optical transceivers in the network as a measure of the network cost. In the reconfigurable case, the number of reconfigurations associated with the evolution of the virtual topology is also minimized as a second optimization criterion. Considering these two objectives, we aim to take into account the trade-off existing between resources (optical transceivers) and reconfiguration costs. On the one hand, the reconfiguration of the virtual topology design along time may involve savings in optical resources since traffic is sent (received) to (from) different nodes at different peak hours. On the other hand, a reconfigurable virtual topology incurs extra costs provoked by the signaling complexity of the traffic adaptation and also by the disruptions in the existing traffic connections.

In this case, we focus on the MH-VTD-R problem assessing the minimization of the number of transceivers achieved via lightpath reconfiguration, against the amount of reconfigurations required. Since the MH-VTD$\mathrm{R}$ problem is clearly NP-hard, like the single traffic matrix VTD problem (integer capacity planning) [6], heuristic algorithms are required to handle large-sized problems. To this aim, we present two heuristic approaches to handle the multihour problem: (i) a Lagrangean Relaxation via Subgradient optimization method (denoted as $L R$ ), and (ii) an algorithm based on a Tabu Search (denoted as TS).

The work presented in this paper is an extension of the works in [27] and [23], where the aforementioned algorithms were initially proposed. In this paper, we expand the algorithm descriptions, specifically elaborating upon on the theoretical steps that support the LR method. Furthermore, we perform a new series of tests, both for synthetically generated traffic and traffic traces from reference topologies, to better assess the behavior and quality of the algorithmic approaches. We also include new results obtained by solving exact MILP formulations for networks of moderate sizes. For cases when the MILP becomes intractable, we compare with lower bounds on the optimal number of transceivers required in the network.

The rest of the paper is organized as follows. Section II presents the state-of-the-art of multihour planning. Section III proposes an application to MH-VTD-R problems of the well-known Lagrangean Relaxation via Subgradient Optimization. Section IV describes a Tabu Search heuristic approach for the MH-VTD-R problem with penalization of reconfiguration. Section VI shows the results of the case studies considered and, finally, Section VII concludes the paper.

\section{RELATED WORK}

Multi-hour network design has been researched for multiple network technologies [7]-[27] (see [10] for a comprehensive survey). If we focus on optical networks, the first investigations of $\mathrm{MH}$ planning were targeted towards the design of virtual topologies in multi-hop networks based on passive stars [7].

However, in the last decade, the interest of the optical community has shifted to lightpath-based transparent optical networks [3],[8],[11]-[27]. Initial investigations on virtual topology reconfiguration consider solely the case of one-time adaptations. In such cases, a change in traffic is known and procedures adapt the existing VTD to this change, without considering periodic (multi-hour) traffic trends [8],[11]-[14]. In all these works, a common objective is to minimize the number of lightpath reconfigurations in the VTD so as to reduce disruptions in the traffic connections.

Recently, other strategies have been attracting the interest of the research community considering periodicity in transparent optical networks planning [3], [15]-[27]. Most such research efforts have been centered on planning in the lower layer, i.e., finding an RWA for a given sequence of virtual topology designs corresponding to $\mathrm{MH}$ traffic. This is based on the Scheduled Lightpath Demand (SLD) model proposed in [15] where the evolution of individual lightpaths is known in advance. The planning problem then consists of finding a set of valid RWA solutions for the input VTDs, optimizing several network performances, such as the number of wavelengths used in the highest loaded fiber link. The SLD model enables more efficient utilization of resources by exploiting the temporal relationship between lightpaths. In [15], a branch and bound algorithm and Tabu Search heuristic were proposed for the Routing and Wavelength Assignment of a set of SLDs. An enhanced Tabu Search algorithm and efficient greedy algorithms for the same problem were proposed in [16]. Fault tolerant RWA was studied in [17] where the authors propose a Simulated Annealing algorithm using channel re-use and back-up multiplexing. Fault-tolerant RWA SLDs under single component failure was considered in [18]. They develop ILP formulations for the problem with dedicated and shared protection. In [19], the authors indicate some drawbacks in the formulations from [18], and give new ILP formulations for survivable service provisioning in networks with wavelength conversion. Their objective is to minimize the number of wavelengthlinks used by primary and secondary paths with guaranteed restoration in case of single failures.

A more general model, called the sliding scheduled traffic model was proposed in [20]. In this model, the set-up and holding times of lightpath demands are known in advance, but they are allowed to slide within a predefined window. Consequently, service provisioning consists not only of solving the RWA problem, but also scheduling demands in time subject to the sliding window constraints with the objective to minimize demand overlap. In [20], they solve the problems subsequently: first tackling scheduling using a demand time conflict reduction algorithm, and then solving RWA with two proposed approaches. Fault tolerant RWA for the sliding scheduled traffic model in networks without wavelength 
conversion was considered in [21]. They also propose a two-phase approach: time conflict resolution followed by RWA. ILP formulations which jointly solve lightpath scheduling and RWA for the sliding scheduled traffic model are given in [22], along with a faster two-step optimization approach for larger problems.

In contrast to the above detailed works, this paper is centered on the upper layer multihour problem, i.e. determining the evolution of the virtual topology from the $\mathrm{MH}$ traffic. To the best of our knowledge, the only work previous in the literature on the MH-VTD-R problem variant is [23] which presents a naïve approach for the reconfigurable problem where the VTDs are independently planned in each time interval.

In the last years, we have been intensively investigating different MH-VTD problem variants presented in [3],[25]-[27]. In [25] a set of MILP formulations is proposed to solve several MH-VTD-NR and MH-VTD-R problem variants minimizing solely the number of transceivers. In [26], a set of Tabu Search based heuristic algorithms solving the previous problems is presented. In [3], we focus on the comparison of a set of variants of the non-reconfigurable problem, with and without flow routing reconfiguration. A set of MILP formulations for the problem and a 3-step algorithm which is based on the concept of traffic domination [28] are proposed.

Finally, in [27] we center on the reconfigurable problem, minimizing both transceiver and reconfiguration cost. We present two families of heuristic algorithms. One family of algorithms is based on the Tabu Search approach from [26], and the other one is based on a decomposition consisting of a Lagrangean Relaxation and a subgradient optimization of the dual problem.

Concerning to the application of similar heuristic approaches to network planning, we have already quoted above some examples of Tabu Search algorithms proposed for optical networks planning ([15], [16]). The Lagrangean Relaxation approach, on the other hand, has been applied previously in [10], [30] to solve multihour capacity design problems in dynamically reconfigurable ATM-based broadband networks. In these works, timefixed modular capacities were assigned to an underlying and given physical topology, whereas time-varying virtual ATM paths were routed over these modular capacities. This problem is equivalent to the MH-VTDNR problem, a problem variant not addressed herein, considering the time-fixed modular capacities as nonreconfigurable lightpaths, and the virtual ATM paths as non-defined higher layer traffic flows. On the contrary, in this work the algorithm is applied to the optical MHVTD-R problem as formulated in Section III. In this MHVTD problem variant, we attempt to minimize the number of optical transceivers and lightpath reconfigurations in a reconfigurable (time-varying) virtual topology.

\section{LAGRANGEAN RELAXATION (LR) APPROACH}

This section describes the application of the wellknown Lagrangean Relaxation via Subgradient Optimization method [29] to multihour problems in optical networks. We refer to this approach as $L R$ in the rest of this paper. This optimization method is based on relaxing the MH-VTD-R problem formulated as a MILP model. Therefore, we will introduce a formal model for the MH-VTD-R considered in this paper before describing the LR approach proposed.

\section{A. MILP Formulation}

In this subsection, we show an exact MILP formulation proposed for the MH-VTD-R problem which penalizes number of lightpath reconfiguration frequency.

Let $N$ be the number of nodes in the network, and $T$ the number of time slots in the multihour traffic. Let $i, j, s, d, n=\{1 \ldots N\}$ be the indices for the nodes, and $t=\{1 \ldots T\}$ be the index for the time slots Note that we assume that the last time interval $t=T$ is followed by the first time interval $t=1$, since traffic is periodic. Let $h^{t}$ denote the traffic matrix at time slot $t$, and $h_{s d}{ }^{t}$ denote the traffic demand (measured in Gbps) from node $s$ to node $d$, during time interval $t$ ). Let $C$ denote the lightpath capacity in Gbps. The cost of each transmitter and receiver is considered equal, and is represented by $c_{1}$. An artificial cost of reconfiguring (setting up or turning down) a lightpath is denoted as $C_{2}$.

The decision variables of the MH-VTD-R problem are:

- $\mathbf{f}=\left(f_{i j}^{s d t}\right) \in[0,1]$. Fraction of the total traffic demand $h_{s d}{ }^{t}$ from node $s$ to node $d$ that is routed on the existing lightpaths from node $i$ to node $j$.

- $\quad \mathbf{p}=\left(p_{i j}{ }^{t}\right)=\{0,1,2, \ldots\}$. Number of lightpaths from node $i$ to node $j$, required during time interval $t$.

- $\mathbf{t x}=t x_{n}=\{0,1,2, \ldots\}$. Number of transmitters installed in node $n$.

- $\quad \mathbf{r x}=r x_{n}=\{0,1,2, \ldots\}$. Number of receivers installed in node $n$.

- $\mathbf{r}^{+}=\left(r_{i j}^{+}\right)=\{0,1,2, \ldots\}$. Number of new lightpaths set up at time $t$ with respect to the number of existing lightpaths at time $t-1$ (or time $T$ if $t=1)$ between the nodes $(i, j)$.

- $\mathbf{r}^{-}=\left(r_{i j}^{-}{ }^{t}\right)=\{0,1,2, \ldots\}$. Number of lightpaths torn down at time $t$ with respect to the number of existing lightpaths at time $t-1$ (or time $T$ if $t=1$ ) between the nodes $(i, j)$

Then, the problem formulation is given by (1).

$\min c_{1} \sum_{n}\left(t x_{n}+r x_{n}\right)+c_{2} \sum_{i, j, t} r^{+t}$

Subject to

$\sum_{s, d}\left\{h_{s d}^{t} \cdot f_{i j}^{s d t}\right\} \leq C \cdot p_{i j}^{t}, \quad i, j=\{1, \ldots, N\}, t=\{1, \ldots, T\}$ 


$$
\begin{aligned}
& \sum_{j} f_{n j}^{s d t}-\sum_{i} f_{i n}^{s d t}=\left\{\begin{array}{l}
1, \text { if } n=s \\
-1, \text { if } n=d, n, s, d=\{1, \ldots, N\}, \\
0 \text { otherwise }
\end{array}\right. \\
& t=\{1, \ldots, T\} \\
& t x_{n} \geq \sum_{j} p_{n j}^{t}, n=\{1, \ldots, N\}, t=\{1, \ldots, T\} \\
& r x_{n} \geq \sum_{i} p_{i n}^{t}, n=\{1, \ldots, N\}, t=\{1, \ldots, T\} \\
& p_{i j}^{t}-p_{i j}^{t-1}=r^{+}{ }_{i j}^{t}-r^{-t}{ }_{i j}^{t}, i, j=\{1, \ldots, N\}, t=\{2 \ldots, T\} \\
& p_{i j}^{1}-p_{i j}^{T}=r^{+}{ }_{i j}{ }^{-} r^{-}{ }_{i j}^{1}, i, j=\{1, \ldots, N\}
\end{aligned}
$$

The objective function (1a) minimizes the total cost of the transmitters and receivers $\left(c_{1}\right)$ and the artificial lightpath reconfiguration cost $\left(c_{2}\right)$. Constraints $(1 \mathrm{~b})$ and (1c) are the standard link capacity and link-flow conservation constraints, respectively. Constraints (1d) and (1e) guarantee that, at any time, the number of transmitters (receivers) installed at a node $n$ is sufficient to establish the decided number of lightpaths originating (terminating) at $n$, respectively. And, finally, constraints (1f) and (1h) links the $\mathbf{r}^{+}$and $\mathbf{r}^{-}$variables with the accounting for the absolute increase and decrease, respectively, in the number of lightpaths between nodes $i, j$, at time $t$.

\section{B. Lagrangean Relaxation via Dual Subgradient Optimization Algorithm}

The Lagrangean Relaxation approach proposed herein uses the same general procedure described in [10] and [29],[30]. We will describe the application of this procedure to the MH-VTD-R problem, but we encourage the interested reader to consult these works for the detailed mathematical foundations about duality concepts.

The philosophy of the method is based on the idea of solving the MILP formulation (1), denoted in the remaining subsection as primal problem, in the dual variable space. The resulting dual problem consists of the maximization of a non-differentiable concave function. Therefore, the problem can be solved by using a standard iterative subgradient algorithm.

The minimization primal problem (1) is transformed into a maximization dual problem by "relaxing" (or "dualizing") a specially chosen subset of primal constraints. This "relaxation" is performed by adding these constraints to the primal objective function (1a) weighted by "Lagrangean multipliers" (also named "dual variables"). Then, the so-obtained Lagrangean Relaxed Problem is still a minimization problem in the primal variable space but parameterized by the dual multipliers. The optimization of this problem generates a value of the dual objective. Since Lagrangean Relaxed Problem is parameterized by the dual multipliers, the value of dual function will depend on the particular values of the multipliers. Consequently, to solve the dual problem, we are interested in finding those dual variables (or multipliers) maximizing the dual function. The exploration of the dual variable space can be carried out through a heuristic subgradient optimization method, since the dual function is concave. In such a method, we jump from a dual solution to other by following the direction of the subgradient vector of the dual function. The newly found values for the dual multipliers are employed to define a new instance of the Lagrangean Relaxed Problem, which is solved in the next iteration to find a new dual function value. Therefore, the overall method is based on successive iterations, each one of them consisting of a minimization step of the Lagrangean Relaxed Problem; and, a maximization step of the Dual Problem via a subgradient optimization. During these steps, the best primal and dual solutions are stored. We must recall from duality theory that the maximal value of best dual solution constitute a lower bound on the optimal primal objective function value. As a consequence, the method provides us a sub-optimality gap along the iterations. Finally, the algorithm finishes when the suboptimality gap falls below a threshold or a maximal number of iterations is reached. In the next paragraphs, we will detail these aspects.

The Lagrangean Relaxed Problem appears when the constraints (1b), (1d) and (1e) are "relaxed" (or "dualized") by adding them (weighted by Lagrangean multipliers) to the objective function (1a) and eliminating them of the total set of constraints of (1b)-(1h). Let $\boldsymbol{\lambda}=$ $\left(\lambda_{i j}{ }^{t}\right), \boldsymbol{\mu}=\left(\mu_{i}^{t}\right)$ and $\boldsymbol{v}=\left(v_{j}^{t}\right)$ be the Lagrangean (or dual) multipliers associated with the constraints (1b), (1d) and (1e), respectively. Then, the Lagrangean function $L(\boldsymbol{\lambda}, \boldsymbol{\mu}$, $\mathbf{v}, \mathbf{f}, \mathbf{p}, \mathbf{t x}, \mathbf{r x}, \mathbf{r}^{+}$) resulting of the relaxation is:

$$
\begin{aligned}
& L(\boldsymbol{\lambda}, \boldsymbol{\mu}, \mathbf{v}, \mathbf{f}, \mathbf{p}, \mathbf{t} \mathbf{x}, \mathbf{r} \mathbf{x}, \mathbf{r}+)= \\
& =c_{1} \sum_{n}\left\{t x_{n}+r x_{n}\right\}+c_{2} \sum_{i, j, t} r_{i j}^{+t}+ \\
& +\sum_{i, j, t} \lambda_{i j}^{t} \cdot\left\{\sum_{s, d}\left\{h_{s d}^{t} \cdot f_{i j}^{s d t}\right\}-C \cdot p_{i j}^{t}\right\}+ \\
& +\sum_{i, t} \mu_{i}^{t} \cdot\left\{\sum_{j} p_{i j}^{t}-t x_{i}\right\}+ \\
& +\sum_{j, t} v_{j}^{t} \cdot\left\{\sum_{i} p_{i j}^{t}-r x_{j}\right\}= \\
& =\sum_{n}\left\{c_{1}-\sum_{t} \mu_{n}^{t}\right\} \cdot t x_{n}+ \\
& +\sum_{n}\left\{c_{1}-\sum_{t} v_{j}^{t}\right\} \cdot r x_{n}+ \\
& +\sum_{t, i, j} \lambda_{i j}^{t} \cdot\left\{\sum_{s, d}\left\{h_{s d}^{t} \cdot f_{i j}^{s d t}\right\}\right\}+ \\
& +c_{2} \sum_{i, j, t} r^{+}{ }_{i j}^{t}+\sum_{t, i, j}\left\{\mu_{i}^{t}+v_{j}^{t}-C \cdot \lambda_{i j}^{t}\right\} \cdot p_{i j}^{t}=
\end{aligned}
$$




$$
\begin{aligned}
& =L_{1}(\boldsymbol{\mu}, \mathbf{t x})+L_{2}(\mathbf{v}, \mathbf{r x})+ \\
& +L_{3}(\boldsymbol{\lambda}, \mathbf{f})+L_{4}\left(\boldsymbol{\lambda}, \boldsymbol{\mu}, \mathbf{v}, \mathbf{r}^{+}, \mathbf{p}\right)
\end{aligned}
$$

And the Lagrangean Relaxed Problem is:

$$
\left.\begin{array}{l}
W(\boldsymbol{\lambda}, \boldsymbol{\mu}, \mathbf{v})= \\
\min _{\mathbf{f}, \mathbf{p}, \mathbf{t}, \mathbf{r}, \mathbf{r}^{+}}\left\{\begin{array}{l}
L\left(\boldsymbol{\lambda}, \boldsymbol{\mu}, \mathbf{v}, \mathbf{f}, \mathbf{p}, \mathbf{t x}, \mathbf{r x}, \mathbf{r}^{+}\right) \\
\text {subject to } \\
\text { constraints (1c), (1f) and (1g) }
\end{array}\right.
\end{array}\right\}
$$

Where $W(\boldsymbol{\lambda}, \boldsymbol{\mu}, \mathbf{v})$ is the dual function, and the dual problem to solve via subgradient optimization is:

$$
\max _{\lambda, \boldsymbol{\mu}, \boldsymbol{v}}\{W(\boldsymbol{\lambda}, \boldsymbol{\mu}, \boldsymbol{v}) \mid \boldsymbol{\lambda} \geq \mathbf{0}, \boldsymbol{\mu} \geq \mathbf{0}, \boldsymbol{v} \geq \mathbf{0}\}
$$

The minimization problem (3) of the Lagrangean function $L\left(\boldsymbol{\lambda}, \boldsymbol{\mu}, \mathbf{v}, \mathbf{f}, \mathbf{p}, \mathbf{t x}, \mathbf{r x}, \mathbf{r}^{+}\right)$can be decoupled into four sets of separate minimization subproblems since the relaxation of the constraints (1b), (1d) and (1e) has broken the dependencies among some primal decision variables. The first and second sets of subproblems are associated to the variables $\mathbf{t x}$ and $\mathbf{r x}$, respectively. The third set of subproblems corresponds to the variables $\mathbf{f}$ solely restrained by the constraints (1c). And, finally, in the fourth set of subproblems the variables $\mathbf{p}, \mathbf{r}^{+}$and $\mathbf{r}^{-}$ remain linked trough the constraints (1f) and (1g).

$$
\begin{aligned}
& W(\boldsymbol{\lambda}, \boldsymbol{\mu}, \mathbf{v})= \\
& =\min _{\mathbf{f}, \mathbf{p}, \mathbf{t x}, \mathbf{r x}, \mathbf{r}^{+}}\left\{\begin{array}{l}
L\left(\boldsymbol{\lambda}, \boldsymbol{\mu}, \mathbf{v}, \mathbf{f}, \mathbf{p}, \mathbf{t x}, \mathbf{r x}, \mathbf{r}^{+}\right) \\
\text {subject to } \\
\text { constraints }(1 \mathrm{c}),(1 \mathrm{f}),(1 \mathrm{~g})
\end{array}\right\}= \\
& =\min _{\mathbf{t} \mathbf{x}}\left\{L_{1}(\boldsymbol{\mu}, \mathbf{t x})\right\}+\min _{\mathbf{r x}}\left\{L_{2}(\mathbf{v}, \mathbf{r x})\right\}+ \\
& +\min _{\mathbf{f}}\left\{\begin{array}{l}
L_{3}(\boldsymbol{\lambda}, \mathbf{f}) \\
\operatorname{subject} \text { to } \\
\operatorname{constraints}(1 \mathrm{c})
\end{array}\right\}+ \\
& +\min _{\mathbf{p}, \mathbf{r}^{+}}\left\{\begin{array}{l}
L_{4}\left(\boldsymbol{\lambda}, \boldsymbol{\mu}, \mathbf{v}, \mathbf{p}, \mathbf{r}^{+}\right) \\
\operatorname{subject} \text { to } \\
\operatorname{constraints}(1 \mathrm{f}),(1 \mathrm{~g})
\end{array}\right\}= \\
& =W_{1}(\boldsymbol{\mu})+W_{2}(\mathbf{v})+W_{3}(\boldsymbol{\lambda})+W_{4}(\boldsymbol{\lambda}, \boldsymbol{\mu}, \mathbf{v})
\end{aligned}
$$

If we analyze these subproblems, we observe that solutions tx $\mathbf{t x}^{*}, \mathbf{r x} *$ and $\mathbf{f}^{*}$ to the first $\left(W_{1}\right)$, second $\left(W_{2}\right)$ and third $\left(W_{3}\right)$ subproblems, respectively, can be easily computed. For each group $(s, d, t)$, the solutions $f_{i j}^{*}$ sdt is the shortest path between node $s$ and $d$ in network with link weights $\lambda_{i j}{ }^{t}$. The solutions $t x^{*}{ }_{n}$ can be trivially obtained by setting:

$$
t x_{n}^{*}=\left\{\begin{array}{l}
U B_{T X}, \text { if } c_{1}<\sum_{t} \mu_{n}^{t} \\
L B_{T X}, \text { if } c_{1} \geq \sum_{t}^{t} \mu_{n}^{t}, n=\{1 \ldots N\}
\end{array}\right.
$$
by:

Whereas, the solutions $r x^{*}$ can be trivially obtained

$$
r x_{n}^{*}=\left\{\begin{array}{l}
U B_{R X}, \text { if } c_{1}<\sum_{t} v_{n}^{t} \\
L B_{R X}, \text { if } c_{1} \geq \sum_{t}^{t} v_{n}^{t}, n=\{1 \ldots N\}
\end{array}\right.
$$

where $U B_{T X}$ and $U B_{R X}$ are upper bounds on the number of transmitters and receivers, respectively. These upper bounds may be artificially large enough values.

Conversely, $L B_{T X}$ (or $L B_{R X}$ ) represents lower bounds on the number of transmitters (or receivers); and, for each node $n$, we can take the minimum number of transmitters (or receivers) that the node $n$ requires, i.e., the largest number of lightpaths required to add (or drop) the total traffic generated by (or targeted to) the node in any time slot $t$. Thus, these lower bounds can be calculated as follows.

$$
\begin{aligned}
& L B_{T X}(n)=\max _{t=1 \ldots T}\left\{\sum_{j=1}^{N}\left\lceil\frac{h_{n, j}^{t}}{C}\right]\right\} \\
& L B_{R X}(n)=\max _{t=1 \ldots T}\left\{\sum_{i=1}^{N}\left\lceil\frac{h_{i, n}^{t}}{C}\right\rceil\right\}
\end{aligned}
$$

Finally, the solution $\mathbf{p}^{*}, \mathbf{r}^{*^{+}}$and $\mathbf{r}^{*^{-}}$are obtained by solving the fourth set of ILP subproblems $W_{4}$ in (5). Although solving these subproblems implies to solve ILP formulations, we must note that they suppose an important reduction on complexity with respect to the original unrelaxed primal one.

Once we have found a solution $\left(\mathbf{f}^{*}, \mathbf{p}^{*}, \mathbf{t} \mathbf{x}^{*}, \mathbf{r x}^{*}, \mathbf{r}^{*^{+}}\right.$, $\left.\mathbf{r}^{*-}\right)$ that minimizes the Lagrangean function $L(\boldsymbol{\lambda}, \boldsymbol{\mu}, \mathbf{v}, \mathbf{f}$, $\mathbf{p}, \mathbf{t x}, \mathbf{r x}, \mathbf{r}^{+}$) (2), the dual function is totally characterized. Further, we must note that dual function is a piecewise linear concave function, since it is the minimum of the linear functions appearing in the solutions of the subproblems. This is the key point of the method. We have transformed a mixed-integer problem into a convex problem (maximization of a concave function) where a local solution is also a global one. On the other hand, the new concave problem is not differentiable (the objective function is piecewise). Thus, the dual space could not be explored with a classical gradient optimization step but with a subgradient one. The subgradient vector $\mathbf{g}$, used as search direction, is computed as follows: 


$$
\begin{aligned}
& \mathbf{g}=\nabla W(\boldsymbol{\lambda}, \boldsymbol{\mu}, \mathbf{v}) ; \\
& \mathbf{g}=\left[\begin{array}{lll}
\mathbf{g}^{1} & \mathbf{g}^{2} & \mathbf{g}^{3}
\end{array}\right] \\
& \mathbf{g}^{1}=\left(g_{i j t}^{1}\right)=\sum_{s, d}\left\{h_{s d}^{t} \cdot f_{i j}^{s d t}\right\}-C \cdot p_{i j}^{t} ; i, j=\{1, \ldots, N\}, t=\{1, \ldots, T\} \\
& \mathbf{g}^{2}=\left(g_{i t}^{2}\right)=\sum_{j} p_{i j}^{t}-t x_{i} ; \quad i=\{1, \ldots, N\}, t=\{1, \ldots, T\} \\
& \mathbf{g}^{3}=\left(g_{j t}^{3}\right)=\sum_{i} p_{i j}^{t}-r x_{j} ; \quad j=\{1, \ldots, N\}, t=\{1, \ldots, T\}
\end{aligned}
$$

Then, the new triple $(\boldsymbol{\lambda}, \boldsymbol{\mu}, \boldsymbol{v})$ in the dual space in the next iteration $k+1$ is updated by the subgradient step as:

$$
\begin{aligned}
& \boldsymbol{\lambda}^{k+1}=\max \left\{\lambda^{k}+s_{k} \cdot \mathbf{g}^{1}, \mathbf{0}\right\} \\
& \boldsymbol{\mu}^{k+1}=\max \left\{\boldsymbol{\mu}^{k}+s_{k} \cdot \mathbf{g}^{2}, \mathbf{0}\right\} \\
& \mathbf{v}^{k+1}=\max \left\{\mathbf{v}^{k}+s_{k} \cdot \mathbf{g}^{3}, \mathbf{0}\right\}
\end{aligned}
$$

where the step size $s_{k}$ is given by :

$$
s_{k}=p \cdot \frac{\left(\bar{W}-W\left(\lambda^{k}, \boldsymbol{\mu}^{k}, \mathbf{v}^{k}\right)\right)}{\left\|\nabla W\left(\lambda^{k}, \boldsymbol{\mu}^{k}, \mathbf{v}^{k}\right)\right\|^{2}}
$$

where $\bar{W}$ is the best dual function value found along the algorithm's iterations and $p$ is parameter initiated to $p_{0}$ value and halved after a certain number of iterations $k_{\mathrm{p}}$ without improvement in the dual objective, without becoming less than a minimal value $p_{\min }$

The new dual multipliers $\left(\lambda^{k+1}, \boldsymbol{\mu}^{k+1}, \mathbf{v}^{k+1}\right)$ are replaced in the Lagrangean function (2), given rise to a new instance of the Lagrangean Relaxed Problem (3) for the $k+1$ iteration. Then, after solving the new Lagrangean Relaxed Problem, a new subgradient optimization step follows. During the algorithm's iterations, we store the best primal and dual solutions found and update the corresponding suboptimality gap from them. The method continues until a stoping rule such as maximum number of iterations $k_{\operatorname{maz}}>k_{\mathrm{p}}$ is reached; or, the suboptimality gap becomes negligible.

Finally, and before concluding this subsection, we must note that the minimal solution $\left(\mathbf{f}^{*}, \mathbf{p}^{*}, \mathbf{t} \mathbf{x}^{*}, \mathbf{r x}^{*}, \mathbf{r}^{*^{+}}\right.$, $\mathbf{r}^{*-}$ ) that optimizes the Lagrangean Relaxed Problem (3) might be not feasible from the point of view of the primal problem (i.e. primal unfeasible), since we have relaxed some constraints in (1). Therefore, we need to find a primal feasible solution (f*, $\left.\mathbf{p}^{\mathbf{p f}}, \mathbf{t} \mathbf{x}^{\mathbf{p f}}, \mathbf{r} \mathbf{x}^{\mathbf{p f}}, \mathbf{r}^{*^{+}}, \mathbf{r}^{+\mathbf{p f}}, \mathbf{r}^{\mathbf{p f}}\right)$. This solution can be generated from the minimal solution $\mathbf{f}^{*}$ following the next steps. Unlike the solutions $\mathbf{p}^{*}, \mathbf{t} \mathbf{x}^{*}$, $\mathbf{r x}^{*}$, and $\mathbf{r}^{+*}$; the solutions $\mathbf{f}^{*}$ is also primal feasible. For this reason, we may use initially $f^{*}$ in (1b) to compute a primal-feasible solution $\mathbf{p}^{\mathbf{p f}}$. Then, this solution $\mathbf{p}^{\mathbf{p f}}$ will be replaced in (1d) and (1e) to derivate primal-feasible $\mathbf{t} \mathbf{x}^{\mathbf{p f}}$ and $\mathbf{r} \mathbf{x}^{\mathbf{p f}}$; and, in (1f) and (1g) to derivate primalfeasible $\mathbf{r}^{+\mathbf{p f}}$ and $\mathbf{r}^{-\mathbf{p f}}$.

\section{TABU SEARCH (TS) APPROACH}

In this section, a Tabu Search heuristic algorithm, denoted as TS in the remainder of this paper, is proposed to address the MH-VTD-R problem with penalization of lightpath reconfiguration. This is an adaptation of the approach presented in [16] to include the minimization of the reconfigurations in the virtual topology. Tabu Search is an iterative meta-heuristic where the exploration of the solution space is controlled by a memory structure called a tabu list. The tabu list stores a certain number of the last visited solutions, prohibiting the search to reconsider them for as long as they remain in the list. With this, we prevent the search from cycling between neighboring solutions around a local optimum.

First, the algorithm starts with an initial current solution. Then, in each iteration, the algorithm explores all the neighboring solutions with respect to the current one, except those memorized in the tabu list. In our approach, we explore a reduced neighborhood to alleviate the computational load. The solutions explored in the neighborhood are computed by a single-time slot MILP formulation, which constraints the number of available transceivers at a given node $n$ in a given time slot $t$. All the neighboring solutions explored in the iteration are evaluated with a fitness function. The best one becomes in the new current solution in the next iteration. After each iteration, the tabu list and the best found solution overall, called the incumbent solution, are updated. The algorithm terminates according to a predefined termination criterion, in our case, the number of iterations without improvement.

\section{A. Solution Computation}

A solution in $T S$ consists of $T$ virtual topology designs, one for each time slot $t=1, \ldots, T$. Each one of these VTDs is computed by the single time slot MILP formulation (14). This formulation receives as input a single traffic matrix and a set of upper bounds on the number of transceivers at each node. It calculates a virtual topology and its corresponding flow routing with the objective to minimize electronic switching, i.e., to minimize the number of lightpaths traversed by a unit of traffic in the network.

The decision variables are:

- $\mathbf{f}=\left(f_{i j}{ }^{s d}\right) \in[0,1]$. Fraction of the total traffic demand $h_{s d}$ from node $s$ to node $d$ that is routed on the existing lightpaths from node $i$ to node $j$.

- $\quad \mathbf{p}=\left(p_{i j}\right)=\{0,1,2, \ldots\}$. Number of lightpaths from node $i$ to node $j$.

The objective function and the set of constraints are described in (14), for the problem associated with a time slot $t=1, \ldots, T$ :

$$
\min \sum_{i, j, s, d} f_{i j}^{s d}
$$

Subject to 


$$
\begin{aligned}
& \sum_{s, d}\left\{h_{s d} \cdot f_{i j}^{s d}\right\} \leq C \cdot p_{i j}, \quad i, j=\{1, \ldots, N\} \\
& \sum_{j} f_{n j}^{s d}-\sum_{i} f_{i n}^{s d}=\left\{\begin{array}{l}
1, \text { if } n=s \\
-1, \text { if } n=d, \\
0 \text { otherwise }
\end{array}\right. \\
& n, s, d=\{1, \ldots, N\} \\
& \sum_{j} p_{n j} \leq U B_{T X}(n), n=\{1, \ldots, N\} \\
& \sum_{i} p_{i n} \leq U B_{R X}(n) n=\{1, \ldots, N\}
\end{aligned}
$$

Constraints (14b) represent the capacity constraints, and equations $(14 \mathrm{c})$ are the flow conservation constraints. Constraints (14d) and (14e) ensure that the number of lightpaths originating (terminating) at a given node, must be below the pre-fixed upper bounds on number of transmitters (receivers) at that node.

The initial solution in TS is obtained by solving the formulation (14) separately for each time slot $t=\{1 \ldots T\}$, setting to infinity the upper bounds on the transceivers per node $U B_{T X}(n)$ (or $\left.U B_{R X}(n)\right)$.

\section{B. Solution Space Exploration}

Herein, we introduce some concepts and notations required for a better understanding of the algorithm description:

- Activity matrices: For a given solution composed of $T$ virtual topologies, we define a $(N x T)$ matrix named Active Transmitters (AT) matrix. $A T(n, t)=\{0,1,2, \ldots\}$ represents the number of transmitters that are active at node $n$ in time slot $t$ in that solution. In other words, a row $n$ shows how the number of active transmitters at node $n$ varies over time. A column $t$ shows the number of active transmitters at all nodes in time slot $t$. The necessary number of transmitters per node is shown as a vector $T(n)=\max (t)\{A T(n, t)\}$, i.e. the maximum element in each row $n$. The total number of transmitters needed in the network corresponding to that solution is $T_{\text {tot }}=\operatorname{sum}(n)\{T(n)\}$. Consider the following example. Suppose there are 3 nodes and 4 time slots, i.e. $\mathrm{N}=3, \mathrm{~T}=4$, with an Active Transmitters matrix of a potential solution as shown below.

$$
\mathbf{A T}=\left(\begin{array}{llll}
3 & 1 & 1 & 2 \\
2 & 2 & 1 & 1 \\
1 & 2 & 0 & 4
\end{array}\right)
$$

Figure 1. An example of an AT matrix.

In this example, value $A T(1,4)=2$ indicates that in the fourth time slot there are 2 transmitters active at node 1 . The number of necessary transmitters per node is: $T(n)=\left[\begin{array}{lll}3 & 2 & 4\end{array}\right]$, while the total number of transmitters required is $T_{\text {tot }}=11$.
We do the same for receivers to get activity matrix Active Receivers (AR), the necessary number of receivers per node $\mathbf{R}(n)$, and the total required receivers $R_{t o t}$.

- Utilization matrices: For a given solution, we define a $(N \times T)$ matrix, which we denote as Utilization of Transmitters (UT) matrix. It is obtained from matrix AT by subtracting from each element in AT, the value of the maximal element in its row except itself. In other words,

$$
U T\left(n_{i}, t_{i}\right)=A T\left(n_{i}, t_{i}\right)-\max _{t \mid t \neq t_{i}}\left(A T\left(n_{i}, t\right)\right)
$$

According to the above definition, the utilization matrix for the previous example is:

$$
\mathbf{U T}=\left(\begin{array}{cccc}
1 & -2 & -2 & -1 \\
0 & 0 & -1 & -1 \\
-3 & -2 & -4 & 2
\end{array}\right)
$$

Figure 2. An example of an UT matrix.

The positive elements in this matrix indicate the number of transmitters that are only used in a single time slot, i.e. are not very efficiently utilized. For example, $U T(3,4)=2$ indicates that in time slot 4 at node 3 , there are 2 transmitters that are only used in this time slot. Intuitively, trying to rearrange these poorly utilized transmitters may lead to better results. We do the same for receivers from $\mathbf{A R}$ to get a matrix Utilization of Receivers (UR).

TS explores the solution space as follows. The heuristic iteratively solves smaller MILP formulations (14) limiting the number of transceivers for independent time intervals in order to jump between neighboring solutions, and, thus, explore the solution space in a directed manner.

After solving (14), without limiting the number of transceivers, for each time slot $t=\{1 \ldots T\}$ to create an initial solution, Tabu Search iterations are run alternating between limiting the total number of transmitters and the total number of receivers until the maximal number of iterations without improvement of the best solution is met. Upon termination, the incumbent (i.e., best visited) solution is deemed the final result. During these iterations, two tabu lists are maintained, realized as FIFO (First In First Out) queues of finite size corresponding to either transmitters (tabu list $T L_{T X}$ ) or receivers (tabu list $T L_{R X}$ ). Each entry in $T L_{T X}$ (or $T L_{R X}$ ) is a node $n$ where the transmitters (or receivers) cannot be further reduced as long as $n$ remains in the list.

In each iteration, we consider neighboring solutions of a current solution to be all those one changed in only one time slot $t$, i.e., solely a virtual topology and a flow routing at a time slot $t$ is different between two neighbors, whereas the VTDs for the remaining time slots are equal between them. Since there are a large number of such solutions, we propose a neighborhood reduction 
technique to consider those solutions more likely to improve the incumbent solution.

Firstly, if the number of transmitters (or receivers) at some node in the current solution is already at its lower bound according to (8) (or (9)), there is no need to consider neighboring solutions with less transmitters or receivers, respectively. If we consider that we are searching for virtual topologies which use the minimum number of transceivers required to carry the offered periodic traffic, reducing the number of transceivers at highly utilized nodes does not seem very useful. A reduction of these transceivers will need compensation in several time slots. Conversely, reducing a transmitter (receiver) at nodes where transmitters (receivers) are used only in one single time slot can more easily be compensated for. In other words, we think eliminating poorly utilized transceivers where feasible should yield better results.

Consequently, we perform a neighborhood reduction as follows. For each node $n=\{1 \ldots N\}$, we choose one time slot $t$ with poorly utilized transmitters, excepting for those nodes forbidden by the tabu list $T L_{T X}$. These pairs $(n, t)$ correspond to those neighboring solutions taken as candidates with respect to transmitters. Thus, the total number of candidates with respect to transmitters is $N$ minus the size of the tabu list $T L_{T X}$. Analogously, we can define candidates with respect to receivers. Note that candidates cannot include nodes at time slots for which the lower bound on transmitters/receivers is reached.

To choose the set of candidates $(n, t)$ with respect to transmitters, we consider only strictly positive, i.e. poorly utilized, elements in UT, for which $A T(n, t)>L B_{T X}(n)$. For each node $n$, we choose randomly one such element which is not forbidden by the tabu list. If there are no positive elements in UT, we choose a random time slot corresponding to one of the elements with zero value zero. For each obtained candidate $(n, t)$, we run the formulation (14) for the traffic matrix at time $t$ but limiting the maximum number of transmitters at node $n$ to $\mathbf{A T}(n, t)-1$. Receivers at node $n$, along with transmitters and receivers at all other nodes, are also limited to their maximal value along time in the current solution. The new virtual topology obtained by solving the formulation (14) replaces the virtual topology at corresponding time slot $t$ in the current solution, giving the new neighboring solution. The same is done to obtain neighbors from candidates $(n, t)$ with respect to receivers, chosen analogously from non-negative elements of $\mathbf{U R}$ for which $A R(n, t)>L B_{R X}(n)$.

In our example, assuming no violation of the lower bound and tabu list constraints, we would have three neighbors with respect to transmitters obtained from candidates $(1,1),(2,1$ or 2$)$ and $(4,3)$, which correspond to elements $U T(1,1), U T(2,1)$ or $U T(2,2)$, and $U T(4,3)$, respectively. If all neighboring solutions in the reduced neighborhood are infeasible in one iteration, the neighborhood is increased to $2 \cdot T \cdot N$ where candidates correspond to all nodes and all time slots for both transmitters and receivers, and with no constraints imposed by the tabu list.
The quality of all the candidates is assessed by means of a fitness function. The best neighboring solution among these candidates, according to the fitness function, becomes the new current solution in the next iteration. The used fitness function is defined as:

$$
c_{1} \sum_{n}\left\{\max _{t} \sum_{j, j \neq n} p_{n j}^{t}\right\}+c_{1} \sum_{n}\left\{\max _{t} \sum_{i, i \neq n} p_{i n}^{t}\right\}+c_{2} \sum_{i, j, t} \mid p_{i j}^{t}-p_{i j}^{t-1}
$$

where $p_{i j}{ }^{t}$ is the number of lightpaths established between nodes $i$ and $j$ at the time slot $t, c_{1}$ is the cost of one transceiver (or receiver), and $c_{2}$ is the cost of one lightpath reconfiguration.

This fitness function aims to minimize both the number transceivers and the lightpath reconfigurations, assuming reconfigurable equipment, i.e., the same transceivers can be used for different lightpaths as long as they are in different time slots. The lightpath reconfigurations are computed as the increases or decreases in the number of lightpaths between consecutive time slots.

We can observe that (16) matches with (1a) expressed in terms of $p_{i j}{ }^{t}$, since solutions constructed by the Tabu Search algorithm in each iteration are derived from single time interval MILP formulations (14) for all $t$.

After each iteration, if the new current solution selected as above is better than the incumbent solution, the incumbent solution is updated accordingly. In addition to this, the tabu lists $T L_{T X}$ and $T L_{R X}$ are updated to include the node $n$ of the best neighbor in the last iteration.

Finally, the Tabu Search terminates when a maximum number of iterations without improvement is reached.

\section{RESUlts}

This section presents the results of extensive tests conducted to assess the performance of the proposed algorithms, compared with those obtained by the exact MILP formulations (in small networks) and lower bounds to the network cost (for larger networks). The algorithms were implemented in the MatplanWDM tool [31] which links to the TOMLAB/CPLEX library [32] to solve the MILP formulations.

Since we follow the assumption of a sufficient number of wavelengths established in Section I, any VTD has a feasible RWA solution. Thus, the optimization problem is independent of the physical network topology. Therefore, the multihour demand is the only input data to the planning problem. Under these considerations, the Lagrangean Relaxation (LR)-based and the Tabu Search (TS)-based approaches are tested and compared for six network traffic data scenarios: a) three small-sized scenarios based on artificial networks; and, b) three larger-sized scenarios based on reference networks brought from literature. In all these scenarios, the multihour traffic consists of 24 matrices used to illustrate hourly fluctuations over the course of a day. All the 
simulations were run on an Intel Core2 Duo CPU P8400 $2.26 \mathrm{GHz}$ processor.

The small non-real network scenarios consist of three networks of $N=\{4,6,8\}$ nodes, respectively. For each network case, five multi-hour sequences were generated randomly using the model described in the equations (17)-(18).

$$
m_{i j}^{t}=b_{i j} \cdot \operatorname{activity}(t) \cdot r f(R), \quad \forall i, j, t .
$$

According to the model, the traffic between two nodes at a given time $m_{i, j}{ }^{t}$ was calculated as the product of three factors. First, factor $b_{i, j}$ gives the $(i, j)$ coordinate of a base traffic matrix computed for the sequence as follows. $80 \%$ of the values in matrix $b$ (randomly chosen) were set to one, while the remaining $20 \%$ were set to two. This is meant to capture the effect of non-uniformities in the generated traffic matrices. Secondly, activity factor activity $(t)$ in equation (17) intends to capture the effect of traffic intensity variation along the day. Our intensity variation scheme is described by equation (18), based on the intensity model presented in [33].

$$
\operatorname{activity}(t)=\left\{\begin{array}{l}
0.1 \text { if } t \in[1,6] \\
1-0.9 \cdot\left(\cos \left(\frac{\bmod (t, T)-6}{18} \cdot \pi\right)\right)^{10} \text { otherwise }
\end{array}\right.
$$

where $t=1, \ldots, T$

Factor $r f(R)$ in (17) is a random value, uniformly distributed over interval $[1-R, 1+R]$. A new independent sample of the $r f(R)$ factor was used for each value $m_{i, j}{ }^{t}$. The purpose of the $r f$ factor is to include a randomness effect in the traffic intensity. In our tests $R$ was set to 0.2 .

Besides the above commented scenarios, three larger scenarios based on more realistic networks are studied: the Abilene $(N=11)$, NSFNET $(N=14)$ and 15-node worldwide networks. In these three cases, the physical topologies span over several time zones in order to investigate the temporal mismatch of the most loaded traffic hour.

In the Abilene network scenario, we used data from a real traffic trace, publicly available at [1]. This data consists of traffic matrices spanning several weeks. Thus, we averaged all values of the trace taken at the same time in the day to obtain a sequence of 24 matrices illustrating typical hourly fluctuations in a day.

For the NSF net case, a unique multi-hour sequence was generated using the aforementioned model described in equations (17)-(18), using as basis matrix $b$, the reference matrix in [34]. As before, $R$ was set to 0.2 .

Finally, the last scenario corresponds to synthetic multi-hour traffic generated for a 15-node worldwide network. The physical topology is described in Table I and Fig. 3. The traffic was generated using the multi-hour model proposed in [33], for which the traffic between two nodes is proportional to the product of the two node populations; the variation of the traffic activity along the day in a node evolves according to expression (18); and, the traffic demand between a pair of nodes depends of the activity of both end nodes.

In all the scenarios, the sequences of traffic matrices were normalized by multiplying them with a normalization factor $(n f)$ given in equation (19). Value $n f$ was calculated such that the average traffic between two nodes in the most loaded time slot is equal to $\rho \cdot C$, where $C$ is the lightpath capacity, and $\rho$ is a traffic load parameter. The values tested in our study were for $\rho=\{0.1,1,10\}$. A value of $\rho=0.1$ corresponds to the case when the average traffic between two nodes in the most loaded time slot is only $10 \%$ of a single lightpath capacity. On the contrary, a value of $\rho=10$ captures cases in which the average traffic between two nodes in the most loaded time slot is the capacity of ten lightpaths.

$$
\begin{aligned}
& h_{i j}^{t}=m_{i j}^{t} \cdot n f(\rho), \quad \forall i, j, t \\
& \text { where } n f(\rho)=\frac{n \cdot(n-1) \cdot \rho \cdot C}{\max _{t}\left\{\sum_{i, j} m_{i j}^{t}\right\}}
\end{aligned}
$$

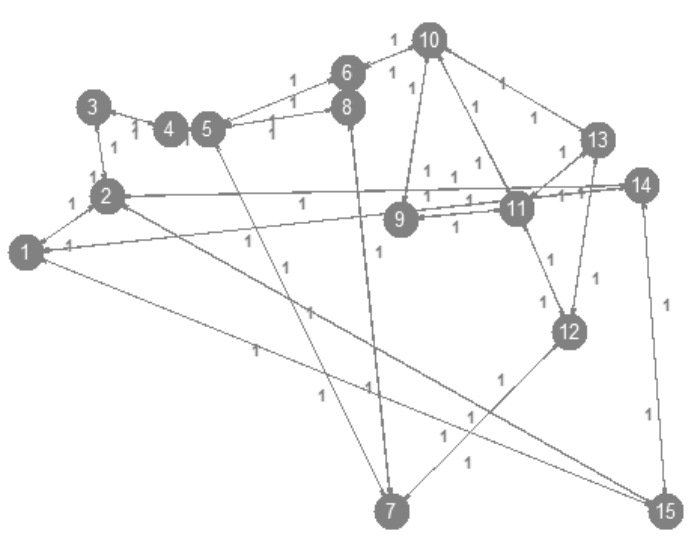

Figure 3. 15-node Worldwide Network Topology.

TABLE I.

DATA OF 15-NODE WORLDWIDE NETWORK TOPOLOGY.

\begin{tabular}{|c|c|r|c|}
\hline Node & Name & Population & Timezone \\
\hline 1 & Honolulu & 378155 & -11 \\
\hline 2 & Los Angeles & 3789981 & -8 \\
\hline 3 & Vancouver & 545671 & -8 \\
\hline 4 & Chicago & 2886251 & -6 \\
\hline 5 & New York & 8084316 & -5 \\
\hline 6 & London & 6638109 & 0 \\
\hline 7 & Cape Town & 2415408 & 1 \\
\hline 8 & Paris & 2125246 & 1 \\
\hline 9 & Athens & 745514 & 2 \\
\hline 10 & Moscow & 10101500 & 3 \\
\hline 11 & New Delhi & 12791458 & 4 \\
\hline 12 & Beijing & 13820000 & 7 \\
\hline 13 & Singapore & 4017733 & 7 \\
\hline 14 & Tokyo & 8134688 & 8 \\
\hline 15 & Sydney & 3997321 & 9 \\
\hline
\end{tabular}


TABLE II.

NUMBER OF TRANSCEIVERS (AND RECONFIGURATIONS) OBTAINED FOR SMALL NETWORK SCENARIOS

\begin{tabular}{|c|c|c|c|c|c|}
\hline $\boldsymbol{N}$ & $\boldsymbol{\rho}$ & LB & MILP & TS & LR \\
\hline \multirow{4}{*}{4} & 0.1 & 8 & $8(1.2)$ & $8(45.6)$ & $21.4(34.8)$ \\
& 1 & 28.2 & $28.2(0.4)$ & $28.4(35.2)$ & $36.6(15.2)$ \\
\cline { 2 - 6 } & 10 & 263.8 & $263.8(7.4)$ & $266.6(237.2)$ & $272.2(235.2)$ \\
\hline \multirow{3}{*}{$\mathbf{6}$} & 0.1 & 12 & $16(1.2)$ & $18.4(96.4)$ & $54.8(152.6)$ \\
& 1 & 71.2 & $71.8(4.4)$ & $71.8(91.6)$ & $91.4(34.8)$ \\
\cline { 2 - 6 } & 10 & 654.2 & $656.6(17.8)$ & $662(616.6)$ & $679.2(612.8)$ \\
\hline \multirow{3}{*}{$\mathbf{8}$} & 0.1 & 16 & $-(-)$ & $31.6(147.8)$ & $105.6(313.4)$ \\
& 1 & 127.6 & $129.8(5.2)$ & $132.6(172.2)$ & $167.2(74.6)$ \\
\cline { 2 - 6 } & 10 & 1199.8 & $1203.2(71.6)$ & $1212.6(1141.8)$ & $1249.8(1143)$ \\
\hline
\end{tabular}

Tables II and III summarize all the performed tests. In Table II, the heuristic approaches are studied for each of the small networks $(N=\{4,6,8\})$ with synthetic traffic. For each network size $N$ and network load $\rho$, we generated five independent sequences of traffic matrices. The columns correspond to:

- LB: lower bounds (LB) on the optimal number of transceivers in the network computed, calculated as:

$$
L B=\sum_{n=1}^{N}\left\{L B_{T X}(n)+L B_{R X}(n)\right\}
$$

where $L B_{T X}$ and $L B_{R X}$ are computed according to (8) and (9).

- MILP: exact solutions of the MILP formulation (1), for those cases where the solver found a solution.

- TS: approximate solutions provided by the TS approach. The stopping criterion was fixed to 20 iterations without improving the best solution found and the size of the tabu list was set to 3, 4 and 5, respectively.

- LR: approximate solutions provided by the LR approach. The total number of iterations was set to 1000 (although the best solution was reached after 50 iterations). The initial and minimal values of stepsize parameter $p$ were fixed to 2 and 0.005 , respectively; and, the maximum number of iterations without improvement to halve $p$ was set to 10 .

In all the approaches, the cost of one transceiver $\left(c_{1}\right)$ was fixed to 1 , whereas the cost of reconfiguring a lightpath $c_{2}$ was set to a sufficiently small fraction $\left(\sim 10^{-5}\right)$ of the cost $c_{1}$, so that the cost of the transceivers dominates the optimization, minimizing the number of transceivers as main objective.

Each cell in Table II shows the average number of transceivers used in the solutions obtained for each of the five sequences corresponding to the same network and load $\rho$. In parenthesis, we include the number of reconfigurations, averaged over the five sequences. The cells marked with (-/-) represent cases where the associated approach was not able to obtain a solution in reasonable time $(250000 \mathrm{~s} \approx 3$ days $)$.
TABLE III.

NUMBER OF TRANSCEIVERS (AND RECONFIGURATIONS) OBTAINED FOR LARGE NETWORK SCENARIOS

\begin{tabular}{|c|c|c|c|c|}
\hline Network & $\boldsymbol{\rho}$ & LB & TS & LR \\
\hline \multirow{2}{*}{$\begin{array}{c}\text { Abilene } \\
(N=11)\end{array}$} & 0.1 & 36 & $52(201)$ & $93(40)$ \\
\cline { 2 - 5 } & 1 & 248 & $260(219)$ & $369(97)$ \\
\hline \multirow{2}{*}{$\begin{array}{c}\text { NSF } \\
(N=14)\end{array}$} & 0.1 & 56 & $235(574)$ & $309(483)$ \\
\cline { 2 - 5 } & 10 & 406 & $424(550)$ & $585(301)$ \\
\hline $\begin{array}{c}\text { Worldwide } \\
(N=15)\end{array}$ & 0.1 & 66 & $104(499)$ & $290(210)$ \\
\cline { 2 - 5 } & 10 & 4920 & $4924(3331)$ & $5134(3092)$ \\
\hline
\end{tabular}

Table III collects the results for the scenarios based on realistic networks: Abilene, NSFNET and the 15-node worldwide network. Herein, for each network and a network load $\rho$, a unique multi-hour traffic sequence is used. The columns of Table III are the same as Table II, excepting the MILP column. In this case, the formulation (1) becomes intractable for the network sizes. On the other hand, the same values as Table I were used for the algorithm parameters, apart from the size of the tabu list in TS. This parameter was set to 7,8 and 9 for the 11node Abilene network, 14-node NSFNET network and the 15-node worldwide networks, respectively.

The results in both tables show that TS outperforms LR in terms of the number of transceivers, while, with respect to reconfiguration frequency, LR obtains similar results for high loads; and, generally better results for medium and low loads. However, these solutions require significantly more transceivers (between 25\% and 234\%) with respect to the TS solutions. These extra transceivers enable a VTD reconfigurable design with fewer reconfigurations. Furthermore, we can observe that the transceiver performance of LR versus TS worsens with load parameter $\rho$.

Finally, the optimality of the TS solutions in terms of the number of transceivers (total transceiver cost) is studied in Tables IV and V for small and large networks, respectively. In Table IV, two sub-optimality gaps are computed with respect to: (i) the optimal MILP solutions for four-, six- and eight-node networks (Gap TS-MILP); and, (ii) the lower bounds (Gap TS-LB) for all the networks. In Table $\mathrm{V}$, the sub-optimality gaps with respect to the lower bounds (Gap TS-LB) are the only one. The analysis of these gaps in the tables confirm that TS is able to provide good approximate solutions for loads $\rho=1$ and $\rho=10$ in general; and, even quasioptimal solutions for $\rho=10$ and for small sized networks. However, for a load of $\rho=0.1$ and large networks $(N \geq 8)$, it is difficult to conclude whether the lower bound is weak or the heuristic solutions are far from optimal, as the suboptimal Gap TS-LB is not negligible and exact MILP solutions were not able to finish for all the cases. Moreover, the only two low load cases $(N=4$, $N=6$ ) where both gaps are available are contradictory, making it hard to assess the weakness of the lower bounds. For $N=4$, the optimal solutions and lower bounds were equal; whereas for $N=6$, the optimal solutions were one third larger than the lower bounds. 
TABLE IV.

OPTIMALITY GAPS OF THE TOTAL TRANSCEIVER COST OF THE TS SOLUTIONS FOR SMALL NETWORK SCENARIOS

\begin{tabular}{|c|c|c|c|}
\hline $\boldsymbol{N}$ & $\boldsymbol{\rho}$ & Gap TS-MILP (\%) & Gap TS-LB (\%) \\
\hline \multirow{3}{*}{4} & 0.1 & $0.00 \%$ & $0.00 \%$ \\
\cline { 2 - 4 } & 1 & $0.70 \%$ & $0.70 \%$ \\
\cline { 2 - 4 } & 10 & $1.05 \%$ & $1.05 \%$ \\
\hline \multirow{3}{*}{6} & 0.1 & $13.04 \%$ & $34.78 \%$ \\
\cline { 2 - 4 } & 1 & $0.00 \%$ & $0.84 \%$ \\
\cline { 2 - 4 } & 10 & $0.82 \%$ & $1.18 \%$ \\
\hline \multirow{3}{*}{8} & 0.1 & - & $49.37 \%$ \\
\cline { 2 - 4 } & 1 & $2.11 \%$ & $3.77 \%$ \\
\cline { 2 - 4 } & 10 & $0.78 \%$ & $1.06 \%$ \\
\hline
\end{tabular}

TABLE V.

OPTIMALITY GAPS OF THE TOTAL TRANSCEIVER COST OF THE TS SOLUTIONS FOR LARGE NETWORK SCENARIOS

\begin{tabular}{|c|c|c|}
\hline Network & $\boldsymbol{\rho}$ & Gap TS-LB (\%) \\
\hline \multirow{2}{*}{$\begin{array}{c}\text { Abilene } \\
(N=11)\end{array}$} & 0.1 & $30.77 \%$ \\
\cline { 2 - 3 } & 1 & $4.62 \%$ \\
\hline \multirow{2}{*}{$\begin{array}{c}\mathrm{NSF} \\
(N=14)\end{array}$} & 0.1 & $0.29 \%$ \\
\cline { 2 - 3 } & 1 & $76.17 \%$ \\
\hline \multirow{2}{*}{$\begin{array}{c}\text { Worldwide } \\
(N=15)\end{array}$} & 0.1 & $4.25 \%$ \\
\cline { 2 - 3 } & 1 & $0.33 \%$ \\
\hline
\end{tabular}

\section{CONCLUSIONS}

In this paper, two heuristic algorithms based on a Lagrangean Relaxation (LR) and a Tabu Search (TS) approach are proposed to solve the Reconfigurable MultiHour Virtual Topology Design (MH-VTD-R) problem assuming two optimization criterions. Both the number of optical transceivers required at the nodes and the number of lightpath reconfigurations necessary to handle the traffic variations over time are minimized as primary and secondary objectives, respectively.

Several test cases are conducted to compare the performance of the two proposed algorithms. The results reveal the superiority of the TS approach with respect to the LR approach in terms of reconfiguration cost. However, LR improves upon TS with respect to reconfiguration performance.

Additionally, the optimality of the heuristic solutions found by the proposed approaches is compared with (i) analytical lower bounds for all the cases; and (ii) exact MILP solutions, for small networks. From these studies, the excellent sub-optimality of the TS solutions in terms of number of transceiver for middle and high traffic loads is demonstrated, while for low loads results are inconclusive due to presumably weak analytical bounds. This sub-optimality, however, comes at the cost of higher reconfiguration frequency.

\section{ACKNOWLEDGMENT}

This research was partially supported by the MICINN/FEDER project grant TEC2010-21405-C0202/TCM (CALM) and MICINN project grant TEC201012250-E (FIERRO). It is also developed in the framework of the projects from Fundación Seneca 00002/CS/08 (FORMA) and "Programa de Ayudas a Grupos de Excelencia de la Región de Murcia" (Plan Regional de Ciencia y Tecnología 2007/2010), and the projects "A Security Planning Framework for Optical Networks (SAFE)," funded by the Unity Through Knowledge Fund (UKF) in Croatia and project 0360362027-1641, funded by the Ministry of Science, Education and Sports, Croatia.

\section{REFERENCES}

[1] J. Berthold, A. A. M. Saleh, L. Blair, and J. M. Simmons, "Optical Networking: Past, Present, and Future," in IEEE J. Lightw. Technol., vol. 26, no. 9, pp.1104-1118, May 2008.

[2] H. Zang, J. P. Jue, and B. Mukherjee, "A Review of Routing and Wavelength Assignment Approaches for Wavelength-Routed Optical WDM Networks," Optical Network Magazine, pp. 47-59, Jan. 2000.

[3] P. Pavon-Marino, R. Aparicio-Pardo, B. Garcia-Manrubia, and N. Skorin-Kapov, "Virtual topology design and flow routing in optical networks under multihour traffic demand," Photonic Network Communications, vol. 19, no. 1, pp. 42-54, Feb. 2010.

[4] TOTEM Project: Toolbox for Traffic Engineering Methods [Online]. $\quad$ Available: http://totem.run.montefiore.ulg.ac.be/datatools.html

[5] S. Uhlig, B. Quoitin, J. Lepropre, S. Balon, "Providing public intradomain traffic matrices to the research community," ACM SIGCOMM Computer Communication Review, vol. 36, no. 1, pp. 83-86, January 2006.

[6] B. Mukherjee, D. Banerjee, S. Ramamurthy, A. Mukherjee, "Some Principles for Designing a Wide-Area WDM Optical Network," IEEE/ACM Transactions on Networking, vol. 4, vo. 5, pp. 684-696, Oct. 1996.

[7] G. Rouskas, M. H. Ammar, "Dynamic Reconfiguration in Multihop WDM Networks," Journal of High Speed Networks, vol. 4, no. 3, pp. 221-238, 1995.

[8] I. Baldine and G. N. Rouskas, "Traffic adaptive WDM networks:A study of reconfiguration issues," Journal of Lightwave Technology, vol. 19, pp. 433-455, Apr. 2001.

[9] G. R. Ash, R. H. Cardwell, and R. P. Murray, "Design and optimization of networks with dynamic routing," Bell Systems Technical Journal, vol. 60, pp. 1787-1820, Oct. 1981.

[10] M. Pioro and D. Medhi, Routing, Flow and Capacity Design in Communication and Computer Networks. San Francisco, CA: Morgan Kaufmann, 2004, pp. 455-474.

[11] A. Gencata, B. Mukherjee, "Virtual-Topology Adaptation for WDM Mesh Networks under Dynamic Traffic," IEEE/ACM Transactions on Networking, vol. 11, no. 2, pp. 236-247, Apr. 2003.

[12] D. Banerjee, B. Mukerjee, "Wavelength-Routed Optical Networks: Linear Formulation, Resource Budgeting Tradeoffs, and a Reconfiguration Study," IEEE/ACM Transactions on Networking, vol. 8, pp. 598-607, 2000.

[13] B. Ramamurthy, A. Ramakrishnan, "Virtual Topology Reconfiguration of Wavelength-Routed Optical WDM Networks," IEEE Globecom 2000, 2000.

[14] K. Liu, C. Liu, J. Pastor, A. Roy, J. Wei, "Performance and Testbed Study of Topology Reconfiguration in IP over Optical Networks," IEEE Transactions on communications, vol. 50, no. 10, pp. 1662-1679, Oct. 2002.

[15] J. Kuri, N. Puech, M. Gagnaire, E. Dotaro, and R. Douville, "Routing and wavelength assignment of scheduled lightpath demands," IEEE Journal on Selected 
Areas in Communications, vol. 21, pp. 1231-1240, Oct. 2003.

[16] N. Skorin-Kapov, "Heuristic algorithms for the routing and wavelength assignment of scheduled lightpath demands in optical networks," IEEE Journal on Selected Areas in Communication, vol. 24, pp. 2-15, 2006.

[17] J. Kuri, N. Puech, and M. Gagnaire, "Diverse routing of scheduled lightpath demands in an optical transport network," in Proc. Design of Reliable Communication Networks (DCRN2003), pp. 69-76, Oct. 2003.

[18] C. V. Saradhi, L. K. Wei, and M. Gurusamy, "Provisioning fault-tolerant scheduled lightpath demands in WDM mesh networks", in Proc. of BroadNets 2004, San Jose, Cal. USA, pp. 150-159, Oct. 2004.

[19] B. Wang, T. Li., C. Xin, X. Zhang, "On survivable service provisioning in WDM networks under a scheduled traffic model", in Proc. of Globecom 2005, St. Louis, MO, USA, pp. 1900-1904, Nov.-Dec. 2005.

[20] B. Wang, T. Li, X. Luo, Y. Fan, and C. Xin, "On service provisioning under a scheduled traffic model in reconfigurable WDM optical networks" in Proc. Of BroadNets 2005, Boston, USA, pp 13-22, Oct. 2005.

[21] C. V. Saradhi, M. Gurusamy, and R. Piesiewicz, "Routing fault-tolerant sliding scheduled traffic in WDM optical mesh networks" in Proc. of BroadNets 2008, London, UK, pp.197-202, Sept. 2008.

[22] A. Jaekel, Y. Chen, "Resource provisioning for survivable WDM networks under a sliding cheduled traffic model", Optical Switching and Networking, Vol. 6, pp. 44-54, 2009.

[23] F. Ricciato, S. Salsano, A. Belmonte, and M. Listanti, "Off-line Configuration of a MPLS over WDM Network under Time-Varying Offered Traffic," IEEE Infocom, pp. 57-65, 2002.

[24] G. Agrawal and D. Medhi, "Lightpath Topology Configuration for Wavelength-Routed IP/MPLS Network for Time-Dependent Traffic", Proc. of IEEE Globecom 2006, San Francisco, Nov-Dec 2006.

[25] B. Garcia-Manrubia, R. Aparicio-Pardo, P. Pavon-Marino, N. Skorin-Kapov, and J. Garcia-Haro, "MILP Formulations for Scheduling Lightpaths under Periodic Traffic," in Proc. 11th International Conference on Transparent Optical Networks, ICTON 2009, Island of São Miguel, Azores, Portugal, June 2009.

[26] N. Skorin-Kapov, P. Pavon-Marino, B. Garcia-Manrubia, and R. Aparicio-Pardo, "Scheduled Virtual Topology Design Under Periodic Traffic in Transparent Optical Networks," in Proc. 6th ICST International Conference on Broadband Communications, Networks and Systems, BroadNets 2009, Madrid, Spain, Sept. 2009.

[27] R. Aparicio-Pardo, P. Pavon-Marino, N. Skorin-Kapov, B. Garcia-Manrubia, and J. Garcia-Haro, "Algorithms for virtual topology reconfiguration under multi-hour traffic," in Proc. 12th International Conference on Transparent Optical Networks, ICTON 2010, Munich, Germany, June 2010, p. We.B1.2.

[28] G. Oriolo, "Domination between traffic matrices," Mathematics of Operations Research, vol. 33, no. 1, pp. 91-96, Feb. 2008.

[29] M. Pioro and D. Medhi, Routing, Flow and Capacity Design in Communication and Computer Networks. San Francisco, CA: Morgan Kaufmann, 2004, pp. 178-184.

[30] D. Medhi and D. Tipper, "Some approaches to solving a multihour broadband network capacity design problem with single-path routing," Telecommunication Systems, vol. 13, no. 2-4, pp. 269-291, Jul. 2000.

[31] MatPlanWDM, Web Site, [Online], Available: http://ait.upct.es/ ppavon/matplanwdm/ Accessed 10 January 2012.
[32] TOMLAB Optimization. Available: http://tomopt.com/. Accessed 10 January 2012.

[33] J. Milbrandt, M. Menth, S. Kopf, "Adaptive Bandwidth Allocation: Impact of Traffic Demand Models for Wide Area Networks," in 19th International Teletraffic Congress (ITC19), Beijing, China, 2005.

[34] R. Ramaswami, K.N. Sivarajan, "Design of Logical Topologies for Wavelength-routed Optical Networks," IEEE J. Select. Areas Commun., vol. 14, no. 5, pp. 840 851, Jun. 1996.

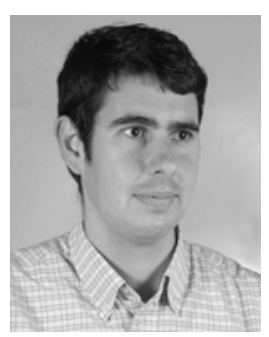

Ramon Aparicio-Pardo received the Engineering degree in Telecommunications and the Ph.D. degree from the Universidad Politécnica de Cartagena (UPCT), Spain, in 2006 and 2011, respectively. He has visited the Networks Lab (University of California at Davis) leaded by Prof. Biswanath Mukherjee from August 2011 to December 2011. His research interests include planning and performance evaluation of optical switching networks.

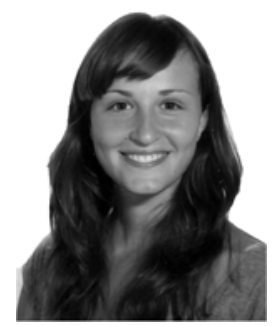

Belen Garcia-Manrubia received the Engineering degree in Telecommunication and the M.Sc. degree from the Technical University of Cartagena (UPCT), Spain, in 2007 and 2008, respectively. She received the $\mathrm{PhD}$. degree from the same university in 2012. Her research interests include planning and performance evaluation of optical networks, especially crosslayer planning under wavelength-routing paradigm.

Nina Skorin-Kapov received the Dipl.-Ing. and Ph.D. degrees in electrical engineering from the University of Zagreb, Zagreb,

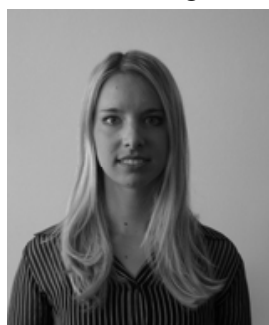
Croatia, in 2003 and 2006, respectively. She has been working as an Assistant Professor at the Department of Telecommunications at the same university since 2008 . She completed a Post-Doctoral Fellowship at Télécom ParisTech (formerly ENST) in Paris, France ,in academic year 2006-7. Her main research interests include optimization of communication networks and network security.

Pablo Pavon-Marino received his Telecommunications Engineering degree from the University of Vigo (Spain) in

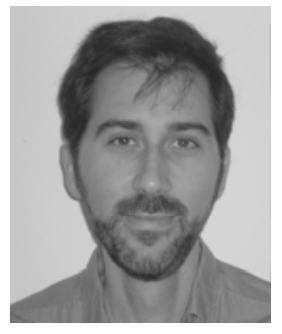
1999. In 2000 he joined the Universidad Politécnica de Cartagena (Spain), where he received his Ph.D. degree in Telecommunications in 2004. In 2010 he received his degree in Mathematics from the UNED University (Spain). He is Associate Professor in the Department of Information Technologies and Communications. His research interests include performance evaluation and optimization of communication networks 